\section{Impacto do uso da água de cisternas na ocorrência de episódios diarréicos na população rural do agreste central de Pernambuco, Brasil}

\section{The impact of the use of water from water- tanks on the occurrence of diarrhea in the rural population of the Central Agreste region of the Brazilian State of Pernambuco}

Carlos Feitosa Luna 1

Ana Maria de Brito 2

André Monteiro Costa 3

Tiago Maria Lapa 4

James A Flint 5

Pasha Marcynuk 6

1-4 Núcleo de Saúde Coletiva. Centro de Pesquisas Aggeu Magalhães. Fundação Oswaldo Cruz. Av. Professor Moraes Rego, s.n. Cidade Universitária. Recife, PE, Brasil. CEP: 50.670-420. E-mail: carlosluna@fiocruz.br

5 Centre for Foodborne, Environmental and Zoonotic Infectious Diseases. Public Health Agency of Canada. Unit 120, 255 Woodlawn Road West, Guelph, Ontario, Canada N1G 5B2

6 Department of Population Medicine, Ontario Veterinary College. University of Guelph. 50 Stone Road East. Guelph, Ontario, Canada.

\section{Abstract}

Objectives: to evaluate the impact of the use of water-tanks on the occurrence of episodes of diarrhea, comparing the number and duration of episodes among the residents of households with and without water-tanks in the same geographical area.

Methods: a longitudinal prospective study, nested in a cross-section study comparing two groups (households with and without water-tanks), was carried out in 21 municipalities in the Central Agreste region of the Brazilian State of Pernambuco, in 2007. Data was gathered over 60 days and included 1,765 individuals. Descriptive analysis was carried out using mixed hierarchical models, Mann-Whitney and Kaplan-Meyer with the level of significance set at $5 \%$.

Results: among the 949 individuals with watertanks, there was a reduction in the risk of the occurrence of episodes of diarrhea of $73 \%$ compared with the 816 individuals without watertanks $(R R=0.27 ; p<0.001)$. The mean number of episodes registered among residents of households without water-tanks was $0.48 \quad(S C=1.17)$, compared with $0.08(S C=0.32)$ among households with water-tanks $(z=-10.26 ; p<0.001)$. The mean during of episodes was 1.5 times greater in households without water-tanks $\left(\chi^{2}=8.99 ; p=0.003\right)$.

Conclusions: the findings of this study point to the importance of access to drinking water for the reduction of disease. The occurrence of diarrhea and its concomitant severity indicators-number of episodes and duration-were consistently higher among residents of households without watertanks.

Key words Potable water, Cistern, Semi-arid, Diarrhea, Pernambuco

\section{Resumo}

Objetivos: avaliar o impacto do uso da água de cisternas na ocorrência de episódios diarréicos comparando o número e a duração de episódios entre moradores de domicílios com e sem cisternas, numa mesma área geográfica.

Métodos: estudo longitudinal prospectivo aninhado a um estudo de corte transversal com dois grupos de comparação (domicílios com e sem cisternas) realizado, em 21 municípios do Agreste Central de Pernambuco, em 2007. O periodo de coleta foi de 60 dias, e incluiu 1765 individuos. Para análise descritiva utilizou-se modelos mistos hierárquicos, Mann-Whitney e Kaplan-Meyer com nivel de significância de 5\%.

Resultados: entre os 949 indivíduos com cisternas, obteve-se uma redução no risco de ocorrência de episódios diarréicos de $73 \%$ quando comparados aos 816 individuos sem cisternas $(R R=0,27 ; p<0.001)$. O número médio de episódios registrados nos residentes de domicílios sem cisternas foi de 0,48 $(D P=1,17)$, contra 0,08 $(D P=0,32)$ nos domicilios com cisternas $(z=-10,26$; $p<0,001)$. A duração média dos episódios foi 1,5 vezes maior nos domicílios sem cisternas $\left(\chi^{2}=8,99 ; p=0,003\right)$.

Conclusões: os achados deste estudo destacam a importância do acesso à água potável na redução de doenças. A ocorrência de diarréia, bem como, seus indicadores de gravidade - número de episódios e duração da diarréia foram consistentemente maiores nos residentes de domicílios sem cisternas.

Palavras-chave Água potável, Cisterna, Semiárido, Diarréia, Pernambuco 


\section{Introdução}

A saúde das populações em países em desenvolvimento é determinada por uma variedade de características relacionadas às condições socioeconômicas, ambientais, nutricionais e de cuidados com a saúde. ${ }^{1,2}$ As doenças diarréicas são um dos principais problemas que afetam a qualidade de vida da população nesses países, especialmente das crianças e idosos, implicando em um aumento de mortalidade e de demanda para a rede de serviços de saúde. 3,4

A água potável é um importante fator no processo de ocorrências de doenças diarréicas, e a forma de acessá-la é determinante na redução de agravos. 5

Globalmente, em 2009, morreram cerca de 8,1 milhões de crianças antes de completarem cinco anos de idade devido a causas evitáveis, com cerca de $77,0 \%$ das mortes associadas à causas neonatais, diarréia, pneumonia e malária. Sendo, diarréia e pneumonia a segunda causa mais comum de morte em crianças menores de cinco anos, ficando atrás apenas das causas neonatais. 6

Constata-se nas últimas décadas, uma forte redução das taxas de mortalidade por diarréia, em todo o mundo, porém essa doença ainda contribui significativamente na mortalidade entre as crianças, com cerca de dois milhões de óbitos por ano.7-9 Além desses óbitos, uma grande parcela da população infantil adoece por diarréia e é acometida por até dez episódios diarréicos ao ano. 10

As reduções nas taxas de mortalidade infantil estão fortemente associadas à melhoria nas condições de vida, melhor acesso aos cuidados médicos, aumento da cobertura vacinal, dos sistemas de esgoto e água potável e no aumento do uso de terapias de re-hidratação oral. ${ }^{11}$

O Brasil acompanha a tendência mundial de redução das taxas de mortalidade por diarréia, nos últimos anos. Esse padrão também tem sido observado na Região Nordeste e, em particular no Estado de Pernambuco, onde o número de óbitos em menores de cinco anos no período de 1996 a 2007 teve uma redução no coeficiente de mortalidade por de 95,18 para 27,49 , aproximadamente $246,23 \% .12$ Em um estudo sobre as doenças diarréicas, $13 \mathrm{em}$ Pernambuco, verificou-se que $24,4 \%$ dos atendimentos ambulatoriais e $35,0 \%$ dos casos de internações em crianças estavam ligados diretamente às diarréias. Nesse mesmo estudo, os autores projetaram uma taxa anual de episódios de diarréia na ordem de 3,9 episódios por criança/ano.

O semi-árido brasileiro é uma região com
$982.563 \mathrm{~km}^{2}, 14$ onde vivem mais de 19 milhões de pessoas. ${ }^{15} \mathrm{~A}$ área é caracterizada por solos rasos e um clima com chuvas concentradas: chuvas fortes (média anual de precipitação de $800 \mathrm{~mm}$ ) que acontecem, principalmente, durante quatro meses do ano. A estrutura sócio-política dessa região é tal que a maioria das terras e dos recursos hídricos são controlados por uma minoria de pessoas que detém o acesso, às fontes d'água. Como resultado, o acesso à água limpa não é apenas uma preocupação prioritária da saúde, mas também uma questão de autonomia sócio-política. 16

O acesso à água potável é considerado como um direito humano fundamental. De acordo com a Organização Mundial da Saúde aproximadamente um bilhão de pessoas ainda não tem acesso à água potável e 2,2 bilhões de pessoas sofrem com doenças veiculadas pela água, anualmente. 17 Devido à escassez de água e acesso restrito, famílias no semiárido brasileiro gastam até 30 horas por mês em busca de água sendo a tarefa de obtenção designada na maioria das vezes a mulheres e crianças. As latas, que contém até 20 litros d'água são transportadas geralmente nas cabeças causando efeitos crônicos negativos, incluindo dor de coluna sendo, esse transporte, na maioria das vezes, feito por crianças e adolescentes aumentando o número de faltas nas salas de aulas decorrente do tempo designado para obtenção de água. 18

A água na região do semi-árido não atinge os níveis de qualidade necessários para o consumo humano, uma vez que as fontes são abertas e suscetíveis à contaminação por esgoto humano, animal e químico.18 Estudo conduzido em Auckland, na Nova Zelândia, destaca que os sistemas de coleta de água de chuva pelo telhado fornecem água potável com boa qualidade físico-química e microbiológica em áreas pobres, além disso ressalta a importância de uma boa estratégia de manutenção desses a fim de minimizar os riscos de contaminação da água. 19

Nesse contexto de dificuldades, em julho de 2003 iniciou-se o Programa de Formação e Mobilização Social para a Convivência com o SemiÁrido: um Milhão de Cisternas Rurais (P1MC) que vem desencadeando um movimento de articulação e de convivência sustentável com o ecossistema do semi-árido, através do fortalecimento da sociedade civil, da mobilização, envolvimento e capacitação das famílias, com uma proposta de educação processual. O objetivo desse programa é beneficiar cerca de cinco milhões de pessoas, em toda região semiárida, disponibilizando água potável para beber e cozinhar, com a construção de cisternas de placas. 
Cada cisterna tem capacidade de armazenar 16 mil litros de água. A água é captada das chuvas, por meio de calhas instaladas nos telhados. As cisternas propiciam às famílias assistidas, mais independência e autonomia na obtenção da água de qualidade, além de melhorar a saúde e a qualidade de vida. ${ }^{20}$

O presente estudo visa comparar a ocorrência, o número e a duração de episódios de diarréia nos moradores residentes em domicílios que possuem cisternas com moradores de domicílios sem cisternas, estratificando por sexo e faixa etária.

\section{Métodos}

Realizou-se um estudo longitudinal prospectivo aninhado a um estudo de corte transversal no qual se comparou a frequência de episódios diarréicos em moradores de uma amostra de domicílios que possuíam cisternas, em relação aos moradores de uma amostra de moradias desprovidas de tal fonte de abastecimento de água.

O estudo foi conduzido na microrregião Agreste Central de Pernambuco, que é composta por 26 municípios e possui uma população de 824.441 indivíduos, dos quais $26 \%$ moradores em área rural. Nessa região, o P1MC está presente em mais de 200 comunidades com cerca de 3000 famílias beneficiadas com cisternas, até maio de 2006 . O número de famílias beneficiadas em cada comunidade varia de cinco até mais de 100 .

Os domicílios com cisternas (construídas pelo $\mathrm{P} 1 \mathrm{MC}$ ) foram selecionados aleatoriamente dos 21 entre os 26 municípios que compõem a microrregião do Agreste Central de Pernambuco. O sorteio foi feito a partir da base de dados fornecida pela Cáritas Diocesana de Caruaru. Foram incluídos no estudo domicílios com pelo menos um morador com idade inferior a cinco anos e com cisternas construídas até $31 / 05 / 2006$, ou seja, com intervalo de tempo superior a um ano entre a sua construção e o início da coleta dos dados do presente estudo.

A amostra de domicílios sem cisterna foi selecionada entre os domicílios da mesma comunidade que preenchiam os critérios de elegibilidade definidos pelo P1MC para o recebimento do benefício (construção da cisterna), porém ainda sem acesso à água proveniente de cisternas Excepcionalmente, quando não havia domicílios nessa situação na mesma comunidade, foram selecionados na(s) comunidade(s) com maior proximidade do município.

Para efeito de análise dos resultados, comparouse a ocorrência de pelo menos um episódio de diarréia nos moradores de domicílios que possuíam cisterna em relação aos domicílios desprovidos da referida fonte de abastecimento, ao final de 60 dias, além do número e duração dos episódios diarréicos.

O tamanho da amostra de domicílios foi calculado considerando-se uma razão de risco de ocorrência de diarréia de pelo menos 1,6 e uma incidência de diarréia de $30 \%$ entre os menores de cinco anos, residentes nos domicílios providos de cisternas. Desta forma, com um nível de significância de $5 \%$ e um poder do teste de $90 \%$, seriam necessários 328 domicílios, com 164 domicílios em cada grupo. Os domicílios foram selecionados sistematicamente a partir da amostra de 391 domicílios com cisterna e 404 domicílios sem cisterna obtida no estudo de corte transversal. Assim a casuística final foi de 197 domicílios sem cisternas e 201 com cisternas com um total de 949 indivíduos residentes em domicílios com cisternas e 816 indivíduos, sem cisternas.

A obtenção dos dados sobre os episódios de diarréia foi realizada durante um período de dois meses. Os indivíduos responsáveis pelos domicílios selecionados receberam uma planilha para acompanhamento diário com anotações de ocorrência de diarréia, número e duração dos episódios de cada morador. As planilhas eram recolhidas a cada 15 dias por um agente comunitário de saúde.

Definiu-se como caso de diarréia, a ocorrência de três ou mais episódios de fezes moles/ líquidas em um período de 24 horas, com ou sem vômito, na ausência de relato sobre a presença de doença de longa duração, uso de medicamentos, abuso de álcool ou gravidez. Os episódios diarréicos foram definidos de acordo com o julgamento do responsável do domicílio, após terem recebido treinamento para a identificação e registro dos casos.

As variáveis independentes, coletadas no momento da aplicação do questionário principal no estudo de corte transversal, foram o domicílio, a localidade, o município, a presença de cisterna, o sexo e a idade dos indivíduos acompanhados.

Realizou-se uma análise descritiva da ocorrência de episódios de diarréia, além do número de episódios e tempo médio de duração, comparando os domicílios com e sem cisternas. Para análise das variáveis categóricas foi aplicado um modelo logístico hierárquico com efeitos mistos. Para a análise hierárquica, as variáveis 'município', 'comunidade' e 'domicílio' foram introduzidas no modelo como fatores aleatórios para corrigir a variância das estimativas e as demais variáveis do estudo foram consideradas como efeitos fixos. Como medida de associação foi utilizado o risco relativo com seus respectivos intervalos de confiança. Para 
comparação do número de episódios diarréicos foi aplicado o teste não-paramétrico de Mann-Whitney. E para comparação do tempo de duração dos episódios foi aplicado o teste Log-rank nas curvas de sobrevida estimadas pelo método de Kaplan-Meyer. Considerou-se um nível de significância de 5\% para a tomada de decisões. O software utilizado foi o R v2.11 e para o ajuste dos modelos mistos foi utilizada a rotina 'glmer'.

Todos os participantes assinaram o termo de consentimento livre e esclarecido após receberem completa explicação sobre o estudo. O trabalho foi revisado e aprovado pela Comissão Nacional de Ética em Pesquisa $\left(\mathrm{N}^{\circ} 470 / 2007\right.$, CAEE: 0081.0.095.000-06) e pelo comitê de ética em pesquisas do Canadá (No REB-2007-0001).

\section{Resultados}

Foram investigados um total de 1765 indivíduos, sendo $51,2 \%$ e $46,7 \%$ do sexo masculino residentes em domicílios com e sem cisternas, respectivamente. Quanto a distribuição etária, nos domicílios com cisternas 3,1\% tem menos de um ano, 20,2\% tem de 1 a 4 anos, $14,0 \%$ de 5 a 9 anos, $19,6 \%$ de 10 a 19 anos, $39,2 \%$ de 20 a 59 anos e $3,9 \%$ com 60 anos ou mais. Enquanto que nos domicílios sem cisternas
$4,9 \%$ tem menos de um ano, $23,2 \%$ tem de 1 a 4 anos, $12,4 \%$ de 5 a 9 anos, $16,4 \%$ de 10 a 19 anos, $40,8 \%$ de 20 a 59 anos e $2,3 \%$ com 60 anos ou mais. Não foram verificadas diferenças significantes e assim podemos dizer que os grupos de comparação são homogêneos em relação à faixa etária e sexo.

A Tabela 1 apresenta a proporção de indivíduos que tiveram pelo menos um episódio de diarréia nos dois grupos de comparação segundo sexo e faixa etária, na qual verifica-se que a cisterna é um fator de proteção na ocorrência de episódios diarréicos. Dessa forma, ter cisterna diminui o risco de ocorrência de episódios diarréicos em 73,0\% $(\mathrm{RR}=0,27$; $p<0,001)$. Quando se estratifica por sexo também se observa diferença entre os domicílios com e sem cisternas, sendo o efeito protetor maior entre os homens $(\mathrm{RR}=0,24 ; p<0,001)$. A análise estratificada por faixa etária revelou diferenças estatisticamente significativas, exceto na faixa de menores de um ano e nos indivíduos com 60 anos ou mais. Entre as faixas com diferenças significantes podemos destacar que a cisterna diminui o risco de ocorrência de diarréia em $84 \%$ nos indivíduos com idades entre 5 e 9 anos $(\mathrm{RR}=0,16 ; p<0,001)$. Entre as demais faixas a chance de ocorrência é muito semelhante evidenciando uma diminuição do risco de acometimento em torno de $70,0 \%$.

\section{Tabela 1}

Distribuição dos moradores da microrregião Agreste Central de Pernambuco avaliado quanto à ocorrência de pelo menos um episódio diarréico no período de 60 dias entre agosto e dezembro de 2007 segundo sexo e faixa etária.

\begin{tabular}{|c|c|c|c|c|c|c|c|c|c|}
\hline & \multicolumn{6}{|c|}{ Domicílios } & \multirow{4}{*}{$\mathbf{z}$} & \multirow{4}{*}{$\begin{array}{l}\text { Risco relativo } \\
\text { (IC95\%) }\end{array}$} & \multirow{4}{*}{$p$} \\
\hline & \multirow{3}{*}{$\mathrm{N}$} & \multicolumn{2}{|c|}{ om cisterna } & \multicolumn{3}{|c|}{ Sem cisterna } & & & \\
\hline & & \multicolumn{2}{|c|}{$\begin{array}{c}\text { Ocorrência } \\
\text { diarréia }\end{array}$} & \multirow[t]{2}{*}{$\mathrm{N}$} & \multicolumn{2}{|c|}{$\begin{array}{l}\text { Ocorrência } \\
\text { diarréia }\end{array}$} & & & \\
\hline & & $\mathrm{n}$ & $\%$ & & $\mathrm{n}$ & $\%$ & & & \\
\hline Ocorrência de diarréia & 949 & 69 & 7,3 & 816 & 199 & 24,4 & $-7,12$ & $0,27(0,19-0,39)$ & $<0,001$ \\
\hline \multicolumn{10}{|l|}{ Sexo } \\
\hline Feminino & 506 & 40 & 7,9 & 398 & 98 & 24,6 & $-5,39$ & $0,31(0,21-0,48)$ & $<0,001$ \\
\hline Masculino & 443 & 29 & 6,5 & 418 & 101 & 24,2 & $-6,32$ & $0,24(0,16-0,38)$ & $<0,001$ \\
\hline \multicolumn{10}{|l|}{ Faixa etária (anos) } \\
\hline$<1$ & 29 & 7 & 24,1 & 40 & 15 & 37,5 & $-0,96$ & $0,64(0,26-1,58)$ & 0,336 \\
\hline 1 a 4 & 192 & 16 & 8,3 & 189 & 59 & 31,2 & $-4,66$ & $0,26(0,15-0,46)$ & $<0,001$ \\
\hline 5 a 9 & 133 & 8 & 6,4 & 101 & 35 & 34,7 & $-4,46$ & $0,16(0,07-0,36)$ & $<0,001$ \\
\hline 10 a 19 & 186 & 12 & 6,5 & 134 & 25 & 18,7 & $-2,17$ & $0,34(0,13-0,90)$ & 0,030 \\
\hline 20 a 59 & 372 & 25 & 6,7 & 333 & 62 & 18,6 & $-4,11$ & $0,34(0,20-0,57)$ & $<0,001$ \\
\hline$\geq 60$ & 37 & 1 & 2,7 & 19 & 3 & 15,8 & $-1,12$ & $0,17(0,01-3,83)$ & 0,265 \\
\hline
\end{tabular}


Os resultados da comparação do número dos episódios de diarréia estão apresentados na Tabela 2. Verifica-se que o número médio de episódios no período de 60 dias foi seis vezes maior entre os moradores de domicílios sem cisternas, com a ocorrência em alguns indivíduos de até dez episódios de diarréia no período analisado $(p<0,001)$.

A análise do número de episódios de diarréia, estratificados por sexo, revela que, entre as mulheres o número médio de episódios de diarréia foi 5,8 vezes maior nos moradores de domicílios sem cisternas $(p<0,001)$. Já entre os indivíduos do sexo masculino o número médio de episódios foi 5,4 vezes maior entre os moradores de domicílios sem cisternas $(p<0,001)$

Já a estratificação por faixa etária revela que o número de episódios é, em média, significativamente maior entre os moradores de domicílios sem cisternas em todas as faixas etárias, exceto nas faixas de menores de um ano e com 60 anos ou mais, onde não foram observadas diferenças significantes. Nesta destacamos que o número médio de episódios de diarréia na faixa etária de 5 a 9 anos foi 8,3 vezes maior entre os moradores de domicílios sem cisternas $(p<0,001)$.

Os resultados da comparação do tempo de duração dos episódios de diarréia estão apresentados na Tabela 3 e as curvas de sobrevida nas Figuras 1 e 2. Nestas verifica-se que o tempo de duração dos episódios diarréicos foi, em média, maior entre os moradores de domicílios sem cisternas $(p=0,003)$. A análise estratificada por sexo revela que, entre os homens essa diferença não foi significativa $(p=0,116)$.

E por fim os resultados da análise do tempo de duração dos episódios diarréicos estratificada por faixa etária revelam que a duração média dos episódios diarréicos foi estatisticamente significante apenas nas faixas etárias de menores de um ano e de 20 a 59 anos. Sendo o tempo médio de duração significativamente maior nas duas faixas nos moradores de domicílios sem cisternas ( $p=0,050 \mathrm{e}$ $p=0,043$, respectivamente).

Distribuição dos moradores da microrregião Agreste Central de Pernambuco avaliado quanto ao número de episódios diarréicos ocorridos no período de 60 dias entre agosto e dezembro de 2007 segundo sexo e faixa etária.

\begin{tabular}{|c|c|c|c|c|c|c|}
\hline & \multicolumn{4}{|c|}{ Domicílios } & \multirow{3}{*}{$\mathbf{z}$} & \multirow{3}{*}{$p$} \\
\hline & \multicolumn{2}{|c|}{ Com cisterna } & \multicolumn{2}{|c|}{ Sem cisterna } & & \\
\hline & $\mathrm{N}$ & $\bar{X} \pm D P$ & $\mathrm{~N}$ & $\bar{X} \pm D P$ & & \\
\hline Número de Episódios & 949 & $0,08 \pm 0,32$ & 816 & $0,48 \pm 1,17$ & $-10,26$ & $<0,001$ \\
\hline \multicolumn{7}{|l|}{ Sexo } \\
\hline Feminino & 506 & $0,09 \pm 0,32$ & 398 & $0,52 \pm 1,28$ & $-7,18$ & $<0,001$ \\
\hline Masculino & 443 & $0,08 \pm 0,32$ & 418 & $0,43 \pm 1,05$ & $-7,33$ & $<0,001$ \\
\hline \multicolumn{7}{|l|}{ Faixa etária (anos) } \\
\hline$<1$ & 29 & $0,28 \pm 0,53$ & 40 & $1,08 \pm 2,13$ & $-1,44$ & 0,151 \\
\hline 1 a 4 & 192 & $0,11 \pm 0,42$ & 189 & $0,67 \pm 1,34$ & $-5,73$ & $<0,001$ \\
\hline 5 a 9 & 133 & $0,08 \pm 0,34$ & 101 & $0,66 \pm 1,24$ & $-5,68$ & $<0,001$ \\
\hline 10 a 19 & 186 & $0,06 \pm 0,25$ & 134 & $0,36 \pm 1,15$ & $-3,48$ & 0,001 \\
\hline 20 a 59 & 372 & $0,07 \pm 0,27$ & 333 & $0,3 \pm 0,83$ & $-4,93$ & $<0,001$ \\
\hline$\geq 60$ & 37 & $0,05 \pm 0,33$ & 19 & $0,16 \pm 0,37$ & $-1,73$ & 0,084 \\
\hline
\end{tabular}


Tabela 3

Distribuição dos moradores da microrregião Agreste Central de Pernambuco avaliado quanto à duração dos episódios diarréicos ocorridos no período de 60 dias entre agosto e dezembro de 2007 segundo sexo e faixa etária.

\begin{tabular}{|c|c|c|c|c|c|c|c|}
\hline & \multicolumn{4}{|c|}{ Domicílios } & \multirow{3}{*}{$\Delta^{* *}$} & \multirow{3}{*}{$t$} & \multirow{3}{*}{$p$} \\
\hline & \multicolumn{2}{|c|}{ Com cisterna } & \multicolumn{2}{|c|}{ Sem cisterna } & & & \\
\hline & $\mathrm{N}$ & $\bar{X} \pm D P$ & $\mathrm{~N}$ & $\bar{X} \pm D P$ & & & \\
\hline Duração dos episódios & 949 & $2,85 \pm 0,23$ & 816 & $4,16 \pm 0,26$ & 1,31 & 11,0 & 0,001 \\
\hline \multicolumn{8}{|l|}{ Sexo } \\
\hline Feminino & 506 & $2,79 \pm 0,31$ & 398 & $4,51 \pm 0,42$ & 1,72 & 70,0 & 0,001 \\
\hline Masculino & 443 & $2,93 \pm 0,34$ & 418 & $3,79 \pm 0,30$ & 0,86 & 39,3 & 0,001 \\
\hline \multicolumn{8}{|l|}{ Faixa etária (anos) } \\
\hline$<1$ & 29 & $2,29 \pm 0,42$ & 40 & $6,40 \pm 1,50$ & 4,11 & 14,3 & 0,001 \\
\hline 1 a 4 & 192 & $3,52 \pm 0,59$ & 189 & $4,79 \pm 0,51$ & 1,27 & 22,4 & 0,001 \\
\hline 5 a 9 & 133 & $3,25 \pm 0,49$ & 101 & $3,51 \pm 0,48$ & 0,26 & 4,06 & 0,001 \\
\hline 10 a 19 & 186 & $2,33 \pm 0,43$ & 134 & $3,28 \pm 0,45$ & 0,95 & 19,1 & 0,001 \\
\hline 20 a 59 & 372 & $2,56 \pm 0,38$ & 333 & $3,81 \pm 0,43$ & 1,25 & 41,0 & 0,001 \\
\hline$\geq 60$ & 37 & $6,00 \pm 0,00$ & 19 & $2,00 \pm 0,58$ & 4,00 & 30,1 & $0,001 *$ \\
\hline
\end{tabular}

*Test t com correção de Welch; **Diferença.

Figura 1

Curvas de sobrevida do tempo total de duração dos episódios diarréicos dos moradores da microrregião Agreste Central de ocorridos no período de 60 dias entre agosto e dezembro de 2007 e estratificado por sexo.

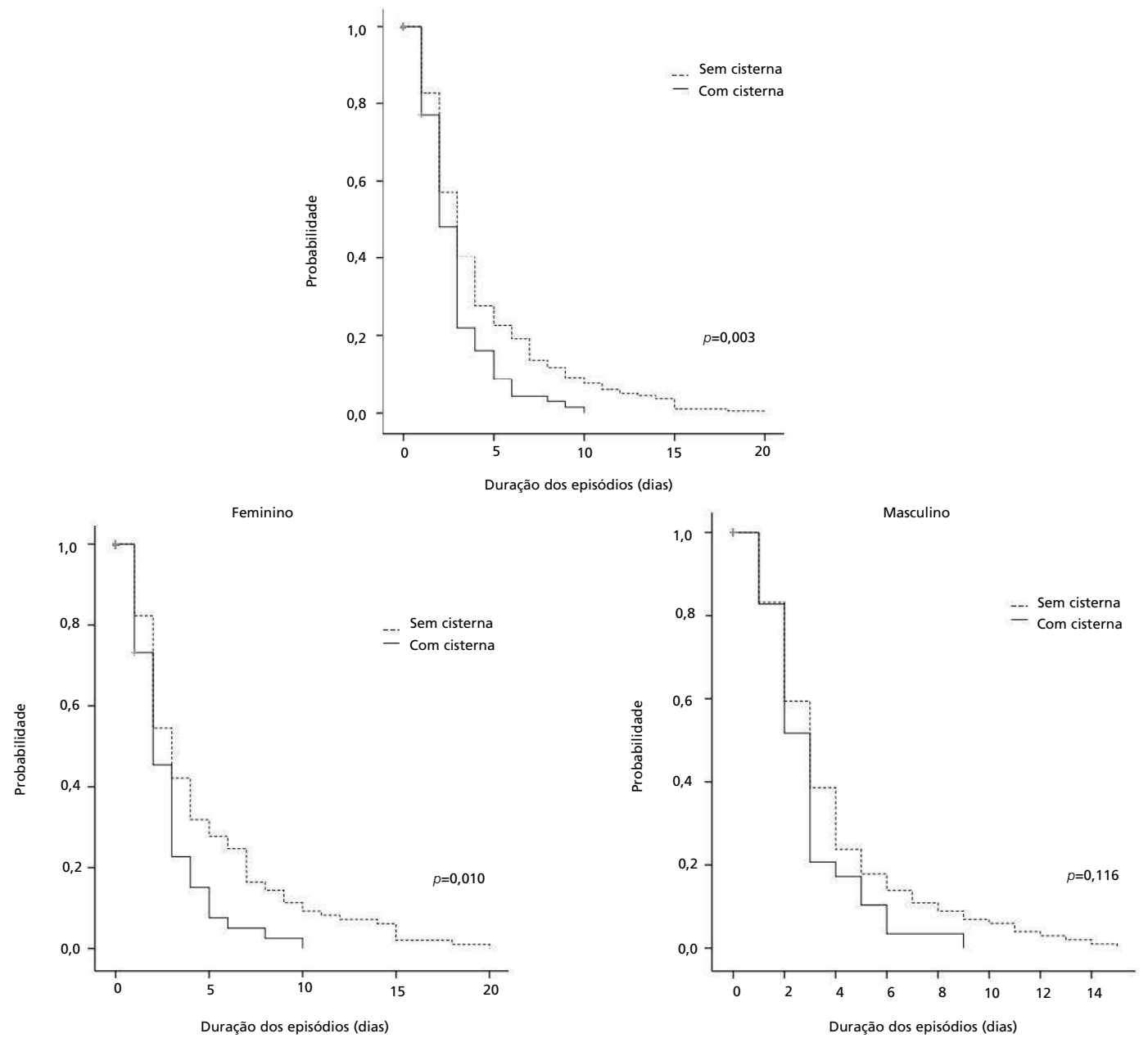


Curvas de sobrevida do tempo de duração dos episódios diarréicos dos moradores da microrregião Agreste Central de ocorridos no período de 60 dias entre agosto e dezembro de 2007 segundo faixa etária.
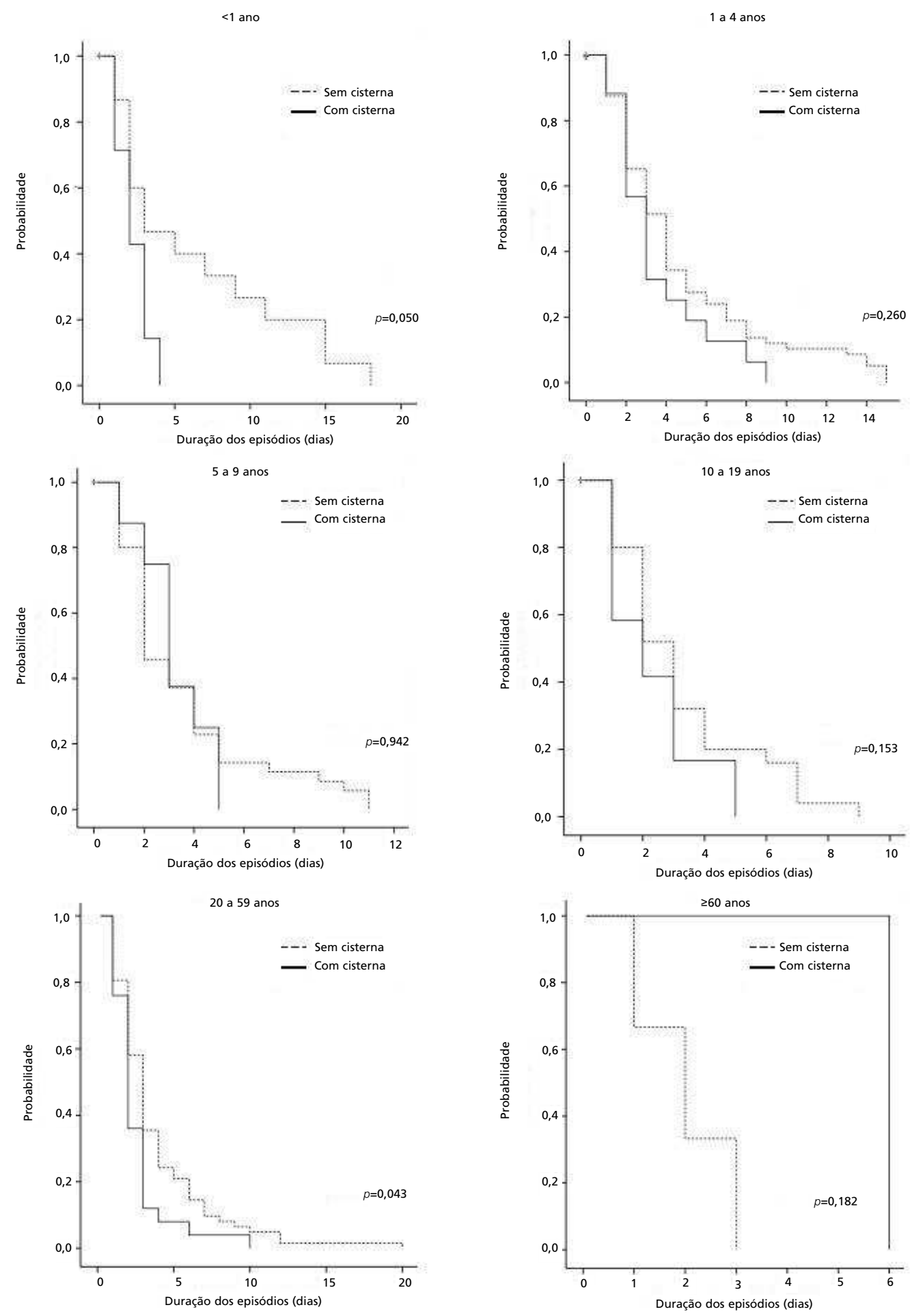


\section{Discussão}

Este estudo revela que a ocorrência de episódios diarréicos é significativamente maior entre moradores de domicílios sem cisterna (24,4\%) quando comparados a moradores de domicílios com cisterna $(7,3 \%)$. As reduções nas proporções ocorrem em ambos os sexo e em indivíduos com idade entre 1 e 59 anos. Quanto aos sexos detectamos uma maior proteção entre os indivíduos do sexo masculino (76\%). O que nos faz pensar que hábitos de vida podem ter determinado esta diferença. Nas faixas etárias de menores de um ano e com 60 ou mais anos, embora as proporções sejam pontualmente maiores entre os indivíduos sem cisternas, o número de indivíduos é muito pequeno. Por essa razão o teste não detectou diferenças significantes entre os dois grupos, uma vez que o tamanho da amostra provoca um considerável aumento na amplitude dos intervalos de confiança.

Ainda em relação à faixa etária temos entre os menores de um ano a presença da amamentação materna, que é um fator de proteção na ocorrência de diarréia em crianças. Embora nesse estudo não tenhamos mensurado a informação sobre a amamentação, sabemos que amamentar por mais de seis meses é um fator de proteção na ocorrência de episódios diarréicos. ${ }^{21} \mathrm{~A}$ verificação de que o número e a duração dos episódios diarréicos são significativamente maiores entre os moradores de domicílios sem cisternas, evidencia que o uso de forma correta da água de chuva captada pelo telhado e armazenada em cisternas pode prover melhor qualidade na saúde da população e ser utilizada como medida de controle e prevenção de ocorrência de diarréia em crianças e adultos, 19,22-25 permitindo assim uma redução das taxas de mortalidade por essa causa, como visto por outros autores. 8,9

A utilização de água de chuva ocorre em vários países de diferentes continentes, onde são oferecidos benefícios para a construção de cisternas para captação da água, como Estados Unidos, Alemanha e Japão. Nesses países, o processo iniciou-se devido a necessidade de retenção de águas pluviais como medida de prevenção no combate a enchentes urbanas. Com o passar do tempo, o risco de escassez e a necessidade de recarga dos subsolos promoveu o aproveitamento dessas águas. ${ }^{26}$

No Quênia um estudo de coorte conduzido na área rural indica que o uso de água de chuva diminui em $30 \%$ o risco de ocorrência de diarréia $(\mathrm{RR}=0,70$; IC $95 \% 0,52-0,95), 24$ já neste estudo foi observado um risco de ocorrência de diarréia $73 \%$ menor em domicílios com cisterna (RR=0,27; IC95\% 0,19-
$0,39)$, o que aponta para o benefício da captação de água de chuva.

Um dos fatores que contribuem para o adoecimento das populações é a falta de cuidados com a higiene das mãos e utensílios usados no contato direto com as águas estocadas, além da ausência de tratamento dessas, o que colabora para a ausência de potabilidade nas águas consumidas, principalmente, em regiões rurais. Um outro fator importante nesse processo, é a composição da água da chuva, que varia de acordo com a localização geográfica, condições meteorológicas, presença de vegetação e carga de poluição. Em áreas de grande densidade de tráfego e nas redondezas industriais, metais pesados, principalmente o chumbo, podem contaminar a água da chuva. Outras substâncias químicas orgânicas presentes em venenos, praguicidas e herbicidas, quando em altas concentrações na atmosfera, também propiciam a contaminação da água. Porém, a contaminação atmosférica da água de chuva é limitada a zonas urbanas e regiões fortemente industrializadas, e mesmo nesses locais é possível obter água de boa qualidade química para diversos usos. 22

Ocorre uma grande variação da qualidade da água de chuva captada durante os primeiros cinco milímetros de precipitação, principalmente após o primeiro milímetro, uma vez que as primeiras águas promovem a limpeza da atmosfera. Mesmo em áreas com elevado grau de poluição atmosférica observouse forte redução na contaminação da água entre o primeiro e o segundo milímetro. 23

A introdução de mecanismos de descarte eficiente dos primeiros milímetros de água faz-se necessário no processo de não-contaminação da água acumulada. Nas cisternas construídas pelo P1MC não foram instalados tais dispositivos, e mesmo assim foram obtidos excelentes resultados na redução da ocorrência de episódios diarréicos. Acredita-se que com a instalação de dispositivos de descarte nas cisternas, possam haver reduções ainda mais significativas nas taxas de ocorrência de episódios diarréicos.

No semi-árido brasileiro existe um forte problema em relação a fontes d'água inadequadas e falta de saneamento, o que aumenta a chance de adoecimento da população por diarréias. 18 Os nossos resultados revelaram maiores chances de acometimento por essa doença nas populações sem acesso à água de boa qualidade. O que aponta para a necessidade de um maior aporte da gestão no sentido de minimizar o problema na região. Em um estudo de coorte realizado na Austrália, 25 com crianças de quatro a seis anos de idade, foi revelado que as taxas de gastroenterite não diferem entre crianças que 
consumiram água de chuva armazenada em tanques quando comparadas às que consumiram água da rede pública, demonstrando que o sistema de captação de água de chuva é uma boa alternativa para suprir carência de abastecimento com água de boa qualidade.

No campo das limitações do estudo pode-se destacar que as observações coletadas não são independentes, uma vez que foram selecionados todos os moradores do domicílio, e por isso os testes estatísticos não utilizam a variância verdadeira no processo de cálculo das estimativas. Para contornar o problema foram introduzidos modelo de efeitos mistos que melhoram as estimativas das variâncias se aproximando um pouco mais das estimativas verdadeiras. Mesmo assim esse modelo ainda não dá conta do processo de correção. Modelos hierárquicos de efeitos mistos baseados na inflação de zeros ou

\section{Referências}

1. Egbuonu L, Starfield B. Child health and social status. Pediatrics. 1982; 69: 550-7.

2. Mosley WH, Chen LC. An analytical framework for the study of child survival in development countries. Popul Develop Rev. 1984; 10 (Supl): 25-48.

3. UNICEF (Fundo das Nações Unidas para a Infância) Situação mundial da infância. Brasília, DF; 2005.

4. Silva VL, Leal MCC, Marino Jacira G, Marques APO. Associação entre carência social e causas de morte entre idosos residentes no Município de Recife, Pernambuco, Brasil. Cad Saúde Pública. 2008; 24: 1013-23.

5. Strina A, Cairncross S, Barreto ML, Larrea C, Prado MS Childhood diarrhea and observed hygiene beharvior in Salvador, Brazil. Trans R Soc Trop Med Hyg. 2005; 157: 1032-38.

6. The United Nations Children's Fund (UNICEF), WHO, The World Bank and the United Nations Population Division. Levels and trends in child mortality, report 2010. New York, NY: UNICEF; 2010.

7. Victora CG. Mortalidade por diarreia: o que o mundo pode aprender com o Brasil? J Pediatr. 2009; 85: 3-5.

8. Moore SR, Lima AA, Schorling JB, Barbosa MS Jr, Soares AM, Guerrant RL. Changes over time in the epidemiology of diarrhea and malnutrition among children in an urban Brazilian shanytown, 1989-1996. Int Journal Infect Dis. 2000; 4: 179-86.

9. Brasil. Ministério da Saúde. Doença diarréica por rotavírus: vigilância epidemiológica e prevenção pela vacina oral de rotavírus. Brasília, DF; 2005.

10. Instituto Adolfo Lutz e Centro de Vigilancia Epidemiologica "Professor Alexandre Vranjac". Diarréia e rotavírus. Rev Saúde Pública. 2004; 38: 844-5.

11. Victora CG, Bryce J, Fontaine O, Monasch R. Reducing deaths from diarrhoea through oral rehydratation therapy. na distribuição binomial negativa poderiam ser utilizados, mas computacionalmente ainda não estão disponíveis. Dificultando ainda mais o processo de análise dos dados.

Mesmo assim, diante de todo o exposto, concluise que a construção de cisternas para armazenamento de água da chuva captada nos telhados, estocando-a para os períodos de estiagem, é uma solução simples, relativamente barata e que pode contribuir significativamente no processo de obtenção de água para o consumo humano em todo o semi-árido brasileiro. Dessa forma, o presente estudo fornece subsídios para a continuação e expansão do P1MC que além de garantir uma forma de aquisição de água com qualidade em área rural, é capaz de reduzir as taxas de ocorrência de episódios de diarréicos, bem como o número e a duração dos episódios.
Bull World Health Organ. 2000; 78: 1246-55.

12. Brasil. Ministério da Saúde/SVS/DASIS - Sistema de informações sobre mortalidade - SIM. [acesso em 10 set 2009]. Disponível em: tabnet.datasus.gov.br/cgi/deftohtm.exe? sim/cnv/obtpe.def.

13. Vasconcelos MJ de OB, Batista-Filho M. Doenças diarréicas em menores de cinco anos no Estado de Pernambuco: prevalência e utilização de serviços de saúde. Rev Bras Epidemiol. 2008; 11: 128-38.

14. Brasil. Ministério da Integração Nacional/ SDR. Relatório final do grupo de trabalho interministerial para redelimitação do semiárido nordestino e do polígono das secas. Ministério de Integração Nacional. Brasília, DF; 2005.

15. IBGE (Instituto Brasileiro de Geografia e Estatística). Censo demográfico de 2000. [acesso em 15 jul 2007]. Disponível em: www.ibge.gov.br.

16. Santos MJ, Araujo LE, Oliveira EM, Silva BB. Seca, precipitação e captação de água de chuva no semi-árido de Sergipe. Eng Ambiental: Pesq Tecnol. 2009; 6: 55-73.

17. Kosek M, Bern C, Guerrant RL. The global burden of diarrhoeal disease, as estimated from studies published between 1992-2000. Bull World Health Organ. 2003; 81: 197-204.

18. Dillingham R, Bern C, Guerrant RL. Childhood stunting: measuring and stemming the staggering costs of inadequate water and sanitation. Lancet. 2004; 363(9403): 94-5.

19. Simmons G, Hope V, Lewis G, Whitmore J, Gao W. Contamination of potable roof-collected rainwater in Auckland, New Zealand. Wat Res. 2001; 35: 1518-24.

20. ANA(Agencia Nacional de Água). Programa de formação e mobilização social para a convivência com o semi-árido: um milhão de cisternas rurais - P1MC. [acesso em 25 set 2006]. Disponível em: http://www.ana.gov.br/GestaoRec Hidricos/UsosMultiplos/arq/PIMC.doc 
21. Silva GAP, Lira PIC, Lima MC. Fatores de risco para doença diarréica no lactente: um estudo caso-controle. Cad Saúde Pública. 2004; 20: 589-95.

22. GROUP RAINDROPS. Aproveitamento de água de chuva. Curitiba; 2002

23. Andrade Neto CO. Segurança sanitária das águas de cisternas rurais. 4o Simpósio Brasileiro de Captação e Manejo de Água de Chuva. Juazeiro, 2003. Anais do 4o Simpósio Brasileiro de Captação e Manejo de Água de Chuva. Juazeiro: ABCMAC,;2003-B.

24. Cunha Melo LR. Variação da qualidade da água de chuva no início da precipitação [dissertação]. Natal: Universidade Federal do Rio Grande do Norte; 2007.

Recebido em 9 de agosto de 2010

Versão final apresentada em 24 de maio de 2011

Aprovado em 20 de junho de 2011
25. Garrett V, Ogutu P, Mabonga P, Ombeki S, Mwaki A, Aluoch G, Phelan M, Quick RE. Diarrhoea prevention in a high-risk rural Kenyan population through point-of-use chlorination, safe water storage, sanitation, and rainwater harvesting. Epidemiol Infect. 2008; 136: 1463-71.

26. Heyworth JS, Glonek G, Maynard EJ, Baghurst PA, FinlayJones J. Consumption of untreated tank rainwater and gastroenteritis among young children in south Australia. Int J Epidemiol. 2006; 35: 1051-8. 